\title{
Eosinophilia With Hepatic Mass and Abnormal Liver Function Tests: An Unusual Presentation of Chronic Myeloid Leukemia
}

\author{
Neeraja Yerrapotu ${ }^{\mathrm{a}}$, Susmitha Edappallath ${ }^{\mathrm{a}}$, Mohammad Jarrar ${ }^{\mathrm{b}}$, Caroline Hamm ${ }^{\mathrm{b}}$, \\ Pat Allevato ${ }^{\mathrm{b}}$, Ali Gabali ${ }^{\mathrm{a}}$, Indryas Woldie ${ }^{\mathrm{b}, \mathrm{c}}$
}

\begin{abstract}
Chronic myeloid leukemia (CML) is a chronic myeloproliferative neoplasm characterized by excess granulocytes at different stages of maturation and the presence of $B C R-A B L 1$ fusion gene or Philadelphia chromosome. Absolute eosinophilia, basophilia, and monocytosis are not uncommon in CML. However, a rare entity called eosinophilic variant of CML (eoCML) can present with eosinophilia without excess neutrophils or basophils. Here, we report a rare and unusual case of eoCML presenting as a liver mass with abnormal liver function tests, which has not been reported in the literature so far.
\end{abstract}

Keywords: Chronic myeloid leukemia; $B C R-A B L$; Hypereosinophilic syndrome; Chronic myelomonocytic leukemia

\section{Introduction}

Chronic myeloid leukemia (CML) is a chronic myeloproliferative neoplasm characterized by excess granulocytes at different stages of maturation and the presence of $B C R-A B L 1$ fusion gene or Philadelphia chromosome. The natural course of CML consists of chronic phase (CP), accelerated phase (AP), and blast phase. Absolute eosinophilia, basophilia, and monocytosis are not uncommon in CML [1]. However, a rare entity called eosinophilic variant of CML (eoCML) can present with eosinophilia without excess neutrophils or basophils. Hypereosinophilic syndrome (HES), characterized by high eosinophil count, differs from eoCML by the absence of the Philadelphia

Manuscript submitted May 29, 2020, accepted June 6, 2020

Published online June 29, 2020

aDepartment of Pathology, Detroit Medical Center, Wayne State University, Detroit, MI 48201, USA

${ }^{b}$ Windsor Regional Cancer Center, Windsor, ON N8W 1L9, Canada

${ }^{\mathrm{c} C o r r e s p o n d i n g ~ A u t h o r: ~ I n d r y a s ~ W o l d i e, ~ W i n d s o r ~ R e g i o n a l ~ C a n c e r ~ C e n t e r, ~}$ 1995 Lens Ave.,Windsor, ON N8W 1L9, Canada.

Email: indriaslw@yahoo.com

doi: https://doi.org/10.14740/jmc3513 chromosome. EoCML typically presents with the involvement of the heart, lungs, and central nervous system. Here, we report a rare and unusual case of eoCML presenting as a liver mass with abnormal liver function tests, which has not been reported in the literature so far.

\section{Case Report}

A 26-year-old male with a past medical history of depression and marijuana use presented to the emergency room (ER) with abdominal pain and dyspepsia for 6 weeks. He had no history of liver disease, alcohol abuse, recent travel, itching, changes in bowel habits, weight loss, fever, or chills. Vital signs in the ER were within normal limits. Physical exam was significant for icteric sclera. There was no mass or tenderness on abdominal exam. Complete blood count was significant for leukocytosis of $25.4 \times 10^{9} / \mathrm{L}$ (reference range $(\mathrm{RR}): 3.6-11 \times 10^{9} / \mathrm{L}$ ) with an absolute eosinophil count of $18.7 \times 10^{9} / \mathrm{L}$ (RR: $0-0.5$ $\left.\times 10^{9} / \mathrm{L}\right)$. His absolute neutrophil count was within normal limits at $3 \times 10^{9} / \mathrm{L}$ (RR: $2-7.5 \times 10^{9} / \mathrm{L}$ ), hemoglobin and platelet count were within normal limits as well. Liver function tests were elevated with aspartate transaminase (AST) of $944 \mathrm{U} / \mathrm{L}$ (RR: <37), alanine transaminase (ALT) of 613 U/L (RR: 11 61), alkaline phosphatase (ALP) of $956 \mathrm{U} / \mathrm{L}(\mathrm{RR}:<126)$, total bilirubin of $199 \mu \mathrm{mol} / \mathrm{L}$ (RR: 0 - 18), conjugated bilirubin of $122 \mu \mathrm{mol} / \mathrm{L}$ (RR: 0 - 10), and LDH of 1,437 U/L (RR: <234). Other blood tests, including prothrombin time (PT)/international normalized ratio (INR), activated partial thromboplastin time (aPTT), lipase, renal function panel were within normal limits.

Extensive testing was performed to identify the etiology of liver injury, which revealed negative viral hepatitis panel, normal ceruloplasmin, alpha-fetoprotein (AFP), anti-mitochondrial antibody (AMA), carcinoembryonic antigen (CEA) and immunoglobulin $\mathrm{G}(\mathrm{IgG})$ levels. The IgE level was elevated at $1,706 \mathrm{kU} / \mathrm{L}$ (RR: $<100 \mathrm{kU} / \mathrm{L})$. Imaging with computed tomography (CT) of the abdomen revealed a hypodense mass in the liver involving the porta hepatis and left lobe measuring at $11.5 \times 5.8 \times 7.5 \mathrm{~cm}$. The spleen was unremarkable and had normal size measuring at $10 \times 4.9 \mathrm{~cm}$.

Peripheral blood smear examination revealed marked leukocytosis (due to eosinophilia) without overt dysplasia. There 


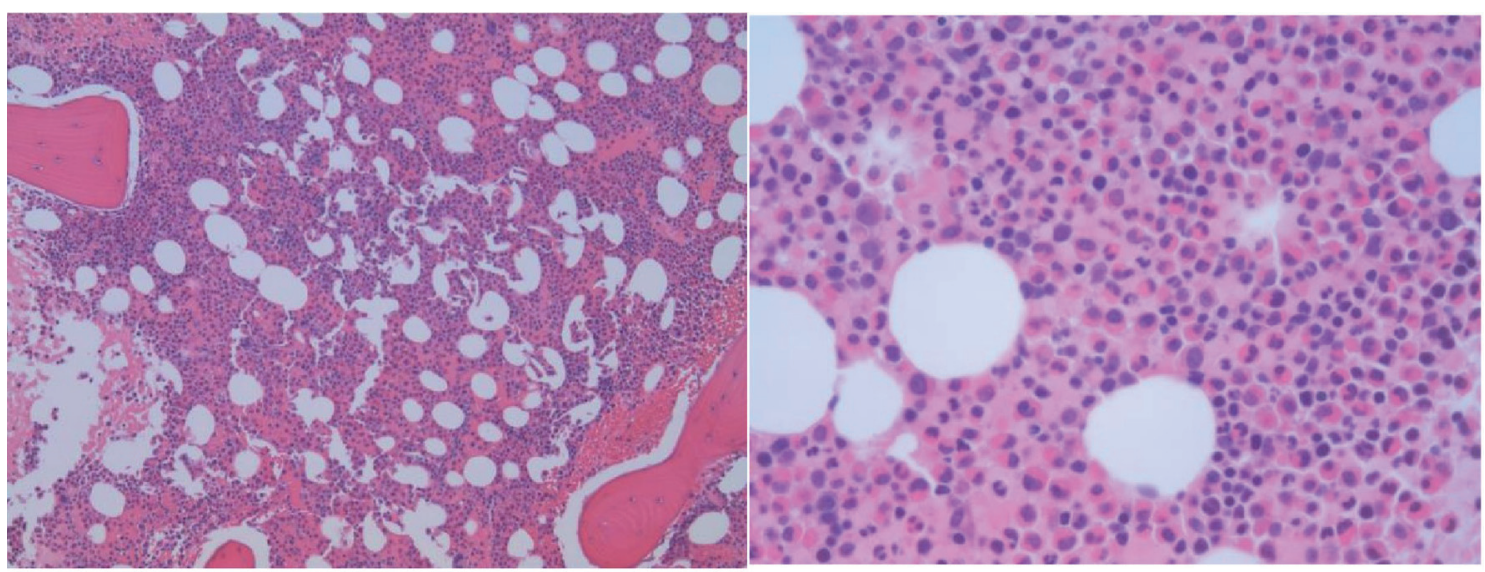

Figure 1. The bone marrow biopsy shows a markedly hypercellular marrow with prominent eosinophilic hyperplasia. No reticulin or collagenous marrow fibrosis is present.

was no increase in blasts or basophils. A concurrent bone marrow examination revealed an increased myeloid/erythroid ratio with marked eosinophilia. There was no morphologic or immunohistochemical evidence of dysplasia, an increase in blasts, nor the presence of B- and T-cell lymphoproliferative neoplasms. Special stains for amyloid deposition and infectious organisms (mycobacteria and fungus) were negative. The hematoxylin and eosin stain of the bone marrow biopsy was shown in Figure 1. Concurrent flow cytometry of the bone marrow aspirate did not reveal any increase in blasts or monocytes or any evidence of aberrant $\mathrm{T}$ cells, monoclonal B cells, and plasma cells.

A core needle biopsy of liver mass was performed, and histopathological examination revealed extensive infiltration with eosinophils associated with a portion of residual hepatic parenchyma (Fig. 2). There was no morphologic or immunohistochemical evidence of an increase in blasts or involvement by B- or T-lymphoproliferative disorders. Additional findings include cholestatic changes, patchy portal, and peri-portal fibrosis.

Cytogenetics performed on the bone marrow speci- men demonstrated three-way variant translocation $\mathrm{t}(2 ; 9 ; 22)$ (q13;q34;q11.2) in five of 16 metaphases examined. Nextgeneration sequencing performed on the bone marrow aspirate identified the presence of a p210 BCR-ABL1 fusion transcript. However, mutations for PCM1-JAK2, PDGFRA/PDGFRB as well as FGFR1 were negative.

Due to a longer turnaround time for cytogenetic studies, the patient was initially placed on steroids for eosinophilia and organ damage, which resulted in severe steroid psychosis. However, once molecular and cytogenetics results were available, the patient was diagnosed with eoCML and started on tyrosine kinase inhibitor (TKI), imatinib at $400 \mathrm{mg}$ daily. The patient achieved hematologic remission within 6 weeks of TKI initiation (except an intermittent and very mild increase in eosinophil count). Within 10 weeks of TKI initiation, his liver enzymes (AST, ALT, and ALP), as well as bilirubin, normalized and he achieved $1.92 \log$ reduction on his quantitative polymerase chain reaction (PCR) for $B C R-A B L(1.2 \% B C R$ $A B L / A B L 1)$ and IS-score of 0.73 which is within the expected response milestone per National Comprehensive Cancer Network (NCCN) guidelines (NCCN guidelines version 1.2019,
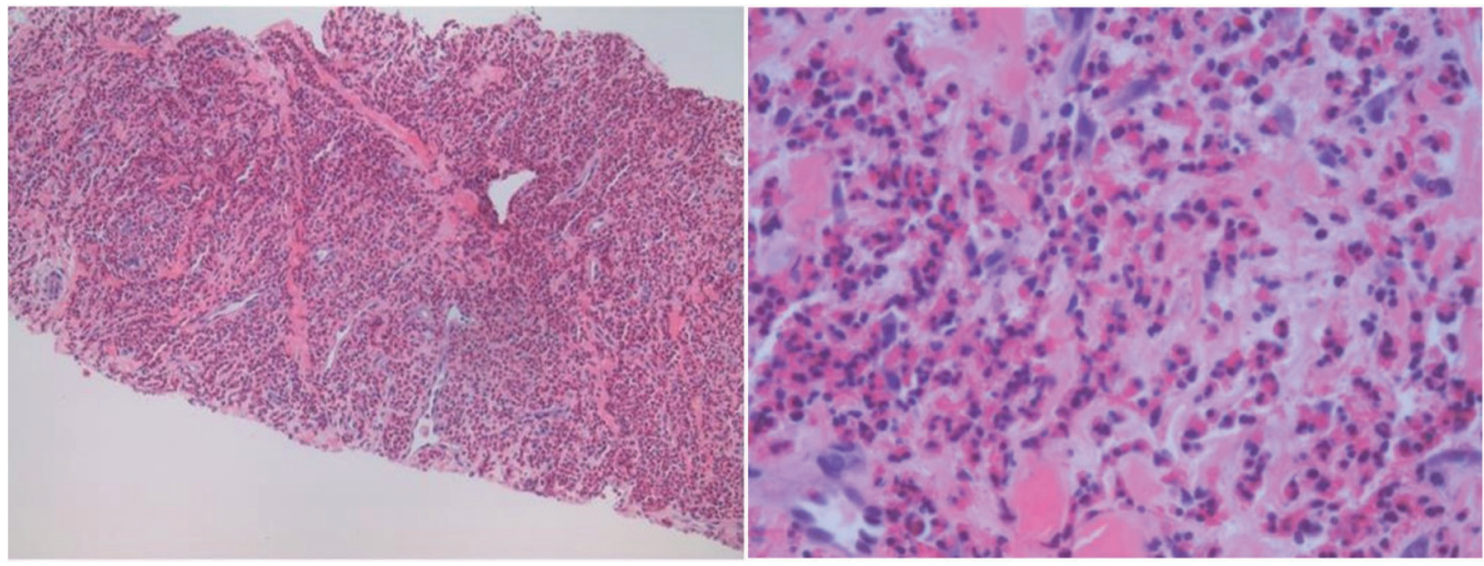

Figure 2. The core biopsy of the liver mass shows an extensive eosinophilic inflammatory cell infiltrate with the destruction of the hepatic parenchyma. Other areas displayed liver lobules and portal tracts infiltrated by numerous eosinophils with degeneration of hepatocytes and cholestasis. 
CML).

Repeat ultrasound imaging performed at 14 weeks following initiation of TKI showed mild hepatomegaly at $18.1 \mathrm{~cm}$ with reduced penetration (possibly mild fatty liver infiltration) but no discrete hepatic mass.

\section{Discussion}

According to the World Health Organization (WHO) classification of tumors of hematopoietic and lymphoid tissue, chronic myelogenous leukemia, $B C R-A B L 1$-positive leukemia is defined as myeloproliferative neoplasm presenting with marked granulocytosis in different stages of maturation with a predominance of myelocytes. Usual clinical findings at presentation include fatigue, malaise, weight loss, and splenomegaly. Absolute basophilia and absolute eosinophilia are the most common supplementary peripheral blood findings in the chronic phase of CML [1]. Cases such as atypical presentation of isolated thrombocytosis without leucocytosis [2-4] with the sole presence of p190 BCR-ABL1 fusion transcript, absolute monocytosis, and features intermediate between CML and chronic myelomonocytic leukemia (CMML) have also been described [5-7].

In the peripheral blood, absolute eosinophilia is defined as $>500$ eosinophils $/ \mathrm{mm}^{3}$. Eosinophilia has been divided into mild $\left(500-1,500 / \mathrm{mm}^{3}\right)$, moderate $\left(1,500-5,000 / \mathrm{mm}^{3}\right)$, and severe $\left(>5,000 / \mathrm{mm}^{3}\right)[8,9]$. The term hypereosinophilia was initially introduced in the 2011 Working Conference on Eosinophil Disorders and Syndromes. It was defined as marked and persistent eosinophilia $\left(>1,500 / \mathrm{mm}^{3}\right)$ and/or the percentage of eosinophils in bone marrow section exceeding $20 \%$ of all nucleated cells and/or the pathologist believes that tissue infiltration by eosinophils is extensive and/or if marked deposition of eosinophil granule proteins is found (in the absence or presence of significant tissue infiltration by eosinophil [9].

Peripheral eosinophilia occurs due to various etiologies, including parasite infestation, hypersensitivity reactions, autoimmune disorders as well as malignancies including hematological malignancies [10].

HES has been divided into three categories: primary, secondary, and idiopathic. In primary/neoplastic HES, eosinophilic expansion occurs due to underlying clonal stem cell, myeloid, or eosinophilic neoplasms [9]. The myeloid and lymphoid neoplasms with eosinophilia and genetic rearrangements are given a special place in the latest 2016 WHO revised classification of hematological tumors. These genetic rearrangements include genes such as $P D G F R-A, P D G F R-B$, FGFR-1, and PCM1-JAK2 [11]. Chronic eosinophilic leukemia, not otherwise specified (CEL, NOS) is a myeloproliferative neoplasm which results in clonal hyperproliferation of eosinophils and eosinophilic precursors in the peripheral blood, bone marrow, and peripheral tissues and is a diagnosis of exclusion. CEL, NOS, is characterized by an absolute eosinophil count of $>1,500 / \mathrm{mm}^{3},<20 \%$ blasts in the peripheral blood and bone marrow and/or presence of a clonal abnormality, and the absence of other clonal abnormalities that results in eosinophilia- $B C R-A B L 1$ fusion, rearrangement of PDGFRA, $P D G$ -
FRB or FGFR1 or PCM1-JAK-2, ETV6-JAK2, or BCR-JAK2 fusion, and other acute and chronic myeloid neoplasms that could present with eosinophilia, like acute myeloid leukemia (AML) with inv(16)(p13.1q22); $\mathrm{t}(1 ; 16)(\mathrm{p} 13.1 ; \mathrm{q} 22), \mathrm{t}(8 ; 21)$ (q22;q22.1), polycythemia vera, essential thrombocythemia, primary myelofibrosis, chronic neutrophilic leukemia, CMML and $B C R-A B L 1$-negative atypical CML [12].

Secondary HES is a reactive, polyclonal proliferation of eosinophils in response to eosinophilic cytokines seen in parasitic infections, certain solid tumors, hematologic neoplasms like T-cell lymphoma, Hodgkin lymphoma, systemic mastocytosis, and lymphoblastic leukemia. Systemic mastocytosis may be associated with the clonal proliferation of eosinophils $[8,9,12]$.

A rare entity called a lymphocytic variant of HES (L-HES) presents as persistent eosinophilia due to cytokine release by aberrant $\mathrm{T}$ cells. It is defined as a clonal proliferation of abnormal memory T cells. Symptoms mimic Sezary syndrome with predominant dermatological manifestations. Less commonly, superficial lymphadenopathy, rheumatological, cardiovascular, and pulmonary involvement are documented. The most common aberrant immunophenotype associated is cluster of differentiation $3(\mathrm{CD} 3)^{-}, \mathrm{CD}^{+} \mathrm{T}$ cells. These cells are proven to release interleukin-5 (IL-5), which eventually causes HES. However, identification of IL-5 levels in the blood is not a routine practice in making this diagnosis. Though there are no definitive diagnostic criteria for L-HES, findings like abnormal immunophenotypes on $\mathrm{T}$ cells like $\mathrm{CD} 3{ }^{-} \mathrm{CD}^{+}, \mathrm{CD}^{+} \mathrm{CD}^{-}$ CD8-, CD4 ${ }^{+} \mathrm{CD}^{-}-$[13], T-cell clonality on PCR studies, rarely occurring cytogenetic abnormalities like 16q breakage, partial 6q, 10q deletion and trisomy 7 [14], increased serum IgE levels, polyclonal hypogammaglobinemia [15], and Epstein-Barr virus (EBV) deoxyribonucleic acid (DNA) levels by PCR are considered in the diagnosis.

Idiopathic HES includes conditions where there is tissue damage as a result of eosinophilia with no known etiology and diagnosed after excluding primary and secondary causes of HES as well as the L-HES. Whereas the term idiopathic hypereosinophilia is used when all the above criteria are met without evidence of tissue damage [8].

Tissue damage by eosinophilia, in general, is thought to be caused via degranulation. Few cases reported include endomyocardial fibrosis and multiple thromboses $[16,17]$. In a case report from India, a patient presented with recurrent superficial thrombophlebitis and eosinophilia secondary to $B C R-A B L$ fusion gene. There was no evidence of splenomegaly similar to our patient [18]. Rare presentations like digital ischemia have been reported as well [19].

In HES, a thorough workup including blood and bone marrow microscopic examination, flow cytometric analysis, cytogenetics, and molecular studies, should be performed to assess clonality after ruling out secondary causes.

The literature has documented very few cases with isolated eosinophilia without neutrophilia or basophilia with associated $B C R-A B L 1$ rearrangement termed as eoCML. EoCML is reported to be a rare presentation of CML, which differs from other forms of HES and CEL, NOS by the presence of $B C R$ $A B L 1$ gene rearrangement and splenomegaly [18-20].

In classic cases of CML, various scoring systems are used for the prediction of prognosis. The most important factor is 
a response to tyrosine kinase inhibitors in all three areas: hematological, cytogenetic, and molecular. Overall, the response for TKI in chronic phase CML is excellent. As the studies have shown, dasatinib and nilotinib have a better and faster response than imatinib. A retrospective cohort analysis performed by Jain et al on patients with chronic phase CML taking imatinib $400 \mathrm{mg}$, imatinib $800 \mathrm{mg}$, dasatinib and nilotinib showed overall 5-year outcomes of failure-free survival (FFS) $70 \%$, transformation-free survival (TFS) $92 \%$, and overall survival (OS) 93\% [21].

There is limited literature published so far and no definitive data about the prevalence of eoCML. Patients with eoCML are treated the same way as classic CML using tyrosine kinase inhibitors. Our patient showed excellent response to Imatinib like other cases mentioned in the literature $[18,19]$.

In our literature review, we have not come across an example of eoCML presenting as liver mass as such with associated abnormal liver function tests, and we hope this unique presentation of CML would provide new information.

\section{Acknowledgments}

None to declare.

\section{Financial Disclosure}

None to declare.

\section{Conflict of Interest}

None to declare.

\section{Informed Consent}

None to declare.

\section{Author Contributions}

NY, MJ, CH, and IW analyzed and interpreted patient data. NY and SE wrote the manuscript with support from IW, PA, and AG. IW, CH, PA, AG, and MJ reviewed and edited the draft manuscript. All authors contributed to the final manuscript.

\section{Data Availability}

Any inquiries regarding supporting data availability of this study should be directed to the corresponding author.

\section{References}

1. Vardiman JW, Melo JV, Baccarani M, et al. Chronic my- elogenous leukaemia, BCR-ABL1 positive. In: WHO Classification of Tumours of Haematopoietic and Lymphoid Tissues. 2017; p. 30-36.

2. Byun YJ, Park BB, Lee ES, Choi KS, Lee DS. A case of chronic myeloid leukemia with features of essential thrombocythemia in peripheral blood and bone marrow. Blood Res. 2014;49(2):127-129.

3. Sora F, Autore F, Chiusolo P, Marietti S, Bayer J, Laurenti L, Giammarco S, et al. Extreme thrombocytosis in chronic myeloid leukemia in the era of tyrosine kinase inhibitors. Leuk Lymphoma. 2014;55(12):2958-2960.

4. Cervantes F, Urbano-Ispizua A, Villamor N, Feliu E, Milla F, Lopez-Guillermo A, Ribera JM, et al. Ph-positive chronic myeloid leukemia mimicking essential thrombocythemia and terminating into megakaryoblastic blast crisis: report of two cases with molecular studies. Leukemia. 1993;7(2):327-330.

5. Verma D, Kantarjian HM, Jones D, Luthra R, Borthakur G, Verstovsek S, Rios MB, et al. Chronic myeloid leukemia (CML) with P190 BCR-ABL: analysis of characteristics, outcomes, and prognostic significance. Blood. 2009;114(11):2232-2235.

6. Pardanani A, Tefferi A, Litzow MR, Zent C, Hogan WJ, McClure RF, Viswanatha D. Chronic myeloid leukemia with p190BCR-ABL: prevalence, morphology, tyrosine kinase inhibitor response, and kinase domain mutation analysis. Blood. 2009;114(16):3502-3503.

7. Melo JV, Myint H, Galton DA, Goldman JM. P190BCR-ABL chronic myeloid leukaemia: the missing link with chronic myelomonocytic leukaemia? Leukemia. 1994;8(1):208-211.

8. Gotlib J. World Health Organization-defined eosinophilic disorders: 2017 update on diagnosis, risk stratification, and management. Am J Hematol. 2017;92(11):12431259.

9. Valent P, Klion AD, Horny HP, Roufosse F, Gotlib J, Weller PF, Hellmann A, et al. Contemporary consensus proposal on criteria and classification of eosinophilic disorders and related syndromes. J Allergy Clin Immunol. 2012;130(3):607-612 e609.

10. Gleich GJ, Leiferman KM. The hypereosinophilic syndromes: still more heterogeneity. Curr Opin Immunol. 2005;17(6):679-684.

11. Barbara BJ, Horny HP. ADM neoplasms with eosinophilia and gene rearrangement I. In: Swerdlow SH, Campo E, Harris NL, et al. HO Classification of Tumours of Haematopoietic and Lymphoid Tissues. 2017; p. 72-79.

12. Barbara BJ, Horny HP. Chronic eosinophilic leukemia. In: Swerdlow SH, Campo E, Harris NL, et al. WHO Classification of Tumours of Haematopoietic and Lymphoid Tissues. 2017; p. 54-56.

13. Lefevre G, Copin MC, Staumont-Salle D, Avenel-Audran M, Aubert H, Taieb A, Salles G, et al. The lymphoid variant of hypereosinophilic syndrome: study of 21 patients with CD3-CD4+ aberrant T-cell phenotype. Medicine (Baltimore). 2014;93(17):255-266.

14. Kitano $\mathrm{K}$, Ichikawa $\mathrm{N}$, Mahbub $\mathrm{B}$, Ueno $\mathrm{M}$, Ito $\mathrm{T}$, Shimodaira S, Kodaira $\mathrm{H}$, et al. Eosinophilia associated with proliferation of $\mathrm{CD}(3+) 4-(8-)$ alpha beta+ $\mathrm{T}$ 
cells with chromosome 16 anomalies. Br J Haematol. 1996;92(2):315-317.

15. Roufosse F, Schandene L, Sibille C, Willard-Gallo K, Kennes B, Efira A, Goldman M, et al. Clonal Th2 lymphocytes in patients with the idiopathic hypereosinophilic syndrome. Br J Haematol. 2000;109(3):540-548.

16. Fauci AS, Harley JB, Roberts WC, Ferrans VJ, Gralnick HR, Bjornson BH. NIH conference. The idiopathic hypereosinophilic syndrome. Clinical, pathophysiologic, and therapeutic considerations. Ann Intern Med. 1982;97(1):78-92.

17. Spry CJ, Take M, Tai PC. Eosinophilic disorders affecting the myocardium and endocardium: a review. Heart Vessels Suppl. 1985;1:240-242.

18. Aggrawal DK, Bhargava R, Dolai TK, Singhal D, Mahapatra M, Rathi S, Bohra B, et al. An unusual presentation of eosinophilic variant of chronic myeloid leukemia
(eoCML). Ann Hematol. 2009;88(1):89-90.

19. Gotlib V, Darji J, Bloomfield K, Chadburn A, Patel A, Braunschweig I. Eosinophilic variant of chronic myeloid leukemia with vascular complications. Leuk Lymphoma. 2003;44(9):1609-1613.

20. Motohashi K, Fujisawa S, Doki N, Kobayashi T, Mori T, Usuki K, Tanaka M, et al. Cytogenetic risk stratification may predict allogeneic hematopoietic stem cell transplantation outcomes for chronic myelomonocytic leukemia. Leuk Lymphoma. 2018;59(6):1332-1337.

21. Jain P, Kantarjian H, Alattar ML, Jabbour E, Sasaki K, Nogueras Gonzalez G, Dellasala S, et al. Long-term molecular and cytogenetic response and survival outcomes with imatinib $400 \mathrm{mg}$, imatinib $800 \mathrm{mg}$, dasatinib, and nilotinib in patients with chronic-phase chronic myeloid leukaemia: retrospective analysis of patient data from five clinical trials. Lancet Haematol. 2015;2(3):e118-128. 